\title{
La medicalización de la vida: hibridaciones ante la dicotomía Naturaleza/Cultura
}

\author{
The medicalization of life: \\ hybrids against the dichotomy Nature/Culture
}

Anahi Sy ${ }^{1}$

${ }^{1}$ Instituto de Salud Colectiva, Universidad Nacional de Lanús. CONICET. Av. 29 de Septiembre 3901, Remedios de Escalada. Buenos Aires Argentina. anahisy@gmail.com

\begin{abstract}
This paper aims to analyze the process of medicalization in current societies, starting from the description of the way in which medicine gradually appropriated various aspects of everyday life that were once part of the life cycle of people. At the theoretical level, we are based on authors such as Descola and Latour, who problematize the dichotomy between Nature and Culture, and propose the need to think from a superior episteme. Methodologically, this theoretical proposal enables an analysis of the medicalization that can illuminate what is hidden in the discourse and biomedical practices: the sociocultural, political and economic processes that are part of these "objects" of Medicine. From this perspective, the presentation of them as scientific facts, objectively isolatable and manipulable by medical science, is in crisis. Thus, our analysis, based on the concept of "quasi-objects" or "hybrids", problematizes such objectification, while providing critical tools to reflect on the medicalization of life in today's societies.
\end{abstract}

Key words Medicalization, Health-Disease process, Medical anthropology
Resumen Este trabajo tiene como objetivo analizar el proceso de medicalización en las sociedades actuales, a partir de la descripción de la forma en que la medicina se ha ido apropiando gradualmente de diversos aspectos de la vida cotidiana que antes formaban parte del ciclo de vida de las personas. Teóricamente, partimos de autores como Descola y Latour quienes problematizan la dicotomía entre Naturaleza y cultura, planteando la necesidad de pensar desde una episteme superadora. Metodológicamente, dicha propuesta teórica habilita un análisis de la medicalización que ilumina lo que se oculta desde el discurso y la prácticas biomédicas: los procesos socioculturales, politicos y económicos que forman parte de tales "objetos" de la medicina. La presentación de los mismos como "hechos cientificos" objetivamente aislables y manipulables por parte de la ciencia médica entra en crisis desde esta perspectiva. Así, nuestro análisis, al partir del concepto de "cuasi -objetos" o "híbridos" problematiza tal objetivación a la vez que aporta herramientas críticas para reflexionar acerca de la medicalización de la vida en las sociedades actuales.

Palabras clave Medicalización, Proceso salud - enfermedad, Antropología médica 


\section{Introducción}

En este trabajo indagamos sobre el proceso mediante el cual diversas esferas de la vida humana se han medicalizado, esto es, patologizado al incorporarse como objetos del discurso y prácticas biomédicas. La atribución de un carácter "natural" o biológico a ciertos objetos por parte de la medicina, habilita la intervención con ciertas prácticas médicas -en nombre de la pretendida objetividad científica- que, al menos en principio, resulta problemática. Para ello partimos de problematizar la forma en que la ciencia médica desde su episteme establece una clara distinción entre naturaleza y cultura al delimitar su "objeto".

Es en este sentido que nos parece relevante para el análisis retomar las propuestas de los antropólogos franceses Philip Descola y Bruno Latour, al plantear la imposibilidad o las dificultades que acarrea la distinción entre lo natural y lo cultural o naturaleza / cultura; el primero al referir a cosmovisiones de los grupos amazónicos $y$, el segundo, al referir a nuestra sociedad, para plantear que la modernidad instala la idea de una clara separación entre naturaleza/cultura que no ha hecho otra cosa más que multiplicar los "hibridos" esto es, "mixtos de naturaleza y cultura", donde tal distinción resulta imposible.

Descola realizó un profundo trabajo de campo etnográfico con los grupos amazónicos Achuar del Ecuador y señala que no se puede establecer en estas culturas una distinción rígida entre el mundo de la naturaleza y el mundo de la cultura; desde ontologías animistas, tal diferenciación resulta inexistente: animales, plantas, paisajes, piedras hasta los astros reciben atributos y características humanas, poseen un alma, son concebidos y tratados como personas ${ }^{1-3}$. En su libro Más allá de la naturaleza y la cultura ${ }^{3}$ plantea lo arbitrario de la episteme científica que separa claramente entre naturaleza y cultura, a partir de la descripción de otras formas de organizar y concebir el mundo que responden a otras epistemes posibles. En ese sentido está planteando que la naturaleza es una construcción cultural y ello supone que la cultura construye a partir de materiales que ella no se ha provisto a sí misma, que ella filtra, codifica, reorganiza o descubre entidades y propiedades primarias, indiferentes a sus intenciones. Es así que, al estar nuestro entorno natural antropizado por todas partes y en grados diversos, su existencia como entidad autónoma ya no es más que una ficción filosófica ${ }^{3}$. Descola va a plantear que este esquema dualista se revela tan inadecuado para pensar nuestras propias prácticas, como para dar cuenta de aquellas sociedades llamadas no modernas, en este sentido señala la imperiosa necesidad de pensar otra episteme ( $y$ en diversas publicaciones cita a Latour como un referente en ese sentido).

Bruno Latour, en su libro Nunca fuimos modernos: ensayos de antropología simétrica ${ }^{4}$, plantea que la división que establece la modernidad entre lo natural y lo cultural resulta ficticia, al examinar diversos temas/problemas actuales entre los cuales podemos citar, a modo de ejemplo, el agujero de ozono o el recalentamiento global, señala que se trata de "híbridos", y los define como humanos, porque se trata de una "obra nuestra", a la vez que naturales, porque "no son producto de nosotros", son locales a la vez que globales. Tales híbridos siempre han estado ahí, si bien desde la constitución moderna han sido negados sistemáticamente por el continuo proceso de "purificación" (El proceso de "purificación" refiere a la creación de dos zonas ontológicas: humanos y no-humamos, estableciendo una división entre un mundo natural y una sociedad con intereses y desafíos previsibles y estables). En este sentido, Latour ${ }^{4}$ analiza las obras de Boyle y Hobbes como aquellas que logran instalar la modernidad, en sus palabras: "inventan nuestro mundo moderno, un mundo en el cual la representación de las cosas por intermedio del laboratorio está disociada para siempre de la representación de los ciudadanos por intermedio del contrato social". Y lleva al extremo el análisis simétrico para plantear que "Boyle no crea simplemente un discurso científico, mientras que Hobbes haría lo mismo para la política; Boyle crea un discurso político donde la política debe ser excluida, mientras que Hobbes imagina una política científica de donde la ciencia experimental debe ser excluida"4. Así, mientras la Constitución moderna (La "Constitución moderna" es el texto común que define el entendimiento que organiza la división de los poderes naturales y políticos, y la separación entre el mundo natural y el mundo social. Esta Constitución ofrece cuatro garantías. 1) Aunque nosotros construyamos la naturaleza, es como si no la construyéramos. 2) Aunque no construyamos la sociedad, es como si la construyéramos. 3) La naturaleza y la sociedad deben ser absolutamente distintas. 4) Dios es alejado para siempre de la doble construcción social y natural, al tiempo que lo deja presentable y utilizable) vuelve invisible, impensable e irrepresentable el trabajo de mediación que reúne a los híbridos; en un hibrido, ni por el lado de la naturaleza ni por el de lo social puede reconocerse las leyes universales 
de las cosas o los derechos imprescindibles de los sujetos (las dos garantías constitucionales de los modernos). Latour, siguiendo a Michel Serres, llama a tales híbridos "cuasi-objetos, porque no ocupan ni la posición de objeto prevista para ellos por la Constitución, ni la de sujetos, y porque es imposible encajonarlos a todos en la posición mediana que los convertiría en una simple mezcla de cosa natural y de símbolo social" y, siguiendo a Levi-Stauss va a decir: se trata de una "íntima fusión por la cual se borran las huellas de los componentes de la naturaleza y de la sociedad -de los que sin embargo dice que se hacen frente 'como en un espejo"'4. Latour menciona un ejemplo interesante, desde esta "antropología simétrica" y que a la vez que lo conecta directamente a los planteos de Levi-Strauss y en particular a Déscola respecto a los Achuar: "También nosotros vinculamos el gesto ínfimo de apretar un aerosol a prohibiciones que atañen al cielo" ${ }^{4}$. $\mathrm{Al}$ respecto, es generalizada la idea de que el uso de aerosoles daña la capa de ozono y, su efecto a la vez que se asocia al calentamiento global, afectaría en lo cotidiano a las personas. Es así que, el agujero de ozono no es solamente un fenómeno natural socialmente construido y discursivo, también está representando a las necesidades y deseos de elementos significativos en el mundo no humano, en este sentido que se puede decir que el comportamiento animista que describe Déscola para los Achuar está también presente en nuestra sociedad.

Consideramos estos aspectos clave a nuestro análisis; también en el proceso de medicalización las distinciones entre ciencia/política o naturale$\mathrm{za} /$ cultura resultan artificiales. Aun cuando desde una episteme de las ciencias de la salud basada en el método Hipotético Deductivo, orientada por la legitimación científico positivista de todo conocimiento se plantee la posibilidad de establecer tal distinción ${ }^{5}$, aquellos objetos de la ciencia médica tampoco corresponden al orden del laboratorio o de la pura naturaleza. Como expresan Castiel y Álvarez Dardet ${ }^{6}$ los discursos acerca de la salud nunca refieren solo a la salud, sino que expresan además formas de pensar y actuar que acompañan un orden económico, político y social que los legitima donde son generados, sustentados y replicados.

La hipótesis que planteamos y buscamos desarrollar en este trabajo, parafraseando a Latour, es que la ciencia médica no solo delimita objetos de la naturaleza, sino que al mismo tiempo crea una política y un discurso político acerca de la sociedad, del mismo modo que la sociedad crea un discurso médico, una naturaleza para los objetos médicos. En este sentido, aparecen como "sujetos a análisis" no solo la medicina, sino también diversos sectores de la sociedad, desde los usuarios del sistema de salud hasta los medios masivos de comunicación, la publicidad y el mercado quienes se apropian, recrean y crean un discurso biomédico.

\section{Medicalización de la vida y sus implicancias}

Al referir a "medicalización de la vida", nos remitimos a desarrollos que provienen del campo de las Ciencias Sociales, Sociología y Antropología Médica y de las Ciencias de la Salud. La medicalización en dichos campos se viene analizando desde fines de los 60: Freidson ${ }^{7}$; Zola ${ }^{8}$; Conrad $^{9}$, Illich ${ }^{10}$, Menéndez ${ }^{11,12}$, Conrad ${ }^{13}$ y Conrad y Schneider ${ }^{14}$.

Ya en la década de 1970, Ivan Illch ${ }^{10}$ ex sacerdote austriaco-americano realiza una crítica a la medicina moderna en su "Némesis de la Medicina" [Nemesis es el nombre de una diosa griega que se encarga de impartir justicia ante la desmesura de los hombres, cuando Illich remite a "nemesis" refiere al castigo que les fue dado a los hombres por "jugar" a ser dioses al intentar manipular la vida y la muerte], señala "la medicina institucionalizada se transformó en una gran amenaza para la salud", describiendo tres formas principales de iatrogénesis: 1- Clínica, que es causada por los propios cuidados de salud, daños por el abuso de las drogas y de las tecnologías médicas más avanzadas; 2- Social, derivada de una creciente dependencia de la sociedad de las drogas, el comportamiento y las medidas prescriptas por las diferentes ramas de las medicina, lo que expropia la salud de cada individuo y de su familia, y disemina en la sociedad el "papel de enfermo", que es el comportamiento pasivo y dependiente de la autoridad médica. 3- cultural, que es la destrucción del potencial cultural de las personas y de las comunidades para lidiar en forma autónoma con la enfermedad el dolor y la muerte. Es decir, aquello que las tradiciones crearon a lo largo de los siglos para enfrentar la vulnerabilidad humana frente a tales contingencias de la vida.

Esto remite a la medicalización, es decir cómo gradualmente procesos, eventos, comportamientos y padecimientos que antes formaban parte de la vida cotidiana o del ciclo de vida de las personas, se han patologizado: por ejemplo las emociones -el duelo por la muerte de un ser querido, el dolor, la angustia o la ansiedad-, 
eventos del ciclo vital -el embarazo, el parto y el nacimiento, la niñez, los cambios hormonales en la adolescencia, la menopausia y el proceso de envejecimiento-, para nombrar sólo algunos. La "construcción" o delimitación que se realiza de tales problemas como plausibles de tratamiento médico es lo que llamamos medicalización.

Es así que la definición de medicalización plantea un nuevo interrogante: ¿¿cuál o qué es un problema médico? pregunta que supone epistémicamente que existen objetos tales que pertenecen al campo de la medicina. Y eso es problemático porque los "objetos" de la medicina no son cosas en el mundo de los objetos, no tienen una pre-existencia hasta que se lo construya como tales. Qué quiere decir esto: no existía un órgano llamado "hígado", "pulmón" o "corazón" hasta que alguien delimitó donde empezaba y terminaba cada uno de tales órganos, los aisló nombrándolos como tales y describió su forma y función diferenciadas. En esta instancia, estamos plateando un posicionamiento teórico, como diría Latour ${ }^{4}$, la modernidad va a instalar la idea que los objetos de las ciencias, creados en laboratorio van a venir a sostener la posibilidad de establecer una distinción entre naturaleza y cultura, entre ciencias naturales y ciencias sociales. Tal distinción no es posible, la medicina toma por objeto de análisis, de estudio y tratamiento un cuerpo, el cuerpo que "enferma", pero ese cuerpo es natural a la vez que modelado, definido y delimitado medicamente, social y culturalmente. $Y$ esto es aún más evidente para las llamadas "enfermedades", su descripción y delimitación no emergen por el descubrimiento de un saber que estaba vedado, sino que se trata de algo que se construye social, histórica y políticamente, y se encuentra sujeto a cambios a lo largo del tiempo. Entonces, la medicalización también supone que algo sea definido en términos médicos, descripto usando un lenguaje médico, entendido desde un marco de referencia médico y tratado desde la intervención médica -aunque no necesariamente todo este proceso ocurre en el laboratorio, ámbito de la ciencia por excelencia, como veremos más adelante. Es importante advertir en este punto que la medicalización describe un proceso; es así que podemos hablar de la medicalización del alcoholismo, la menopausia, la disfunción eréctil o el síndrome de hiperactividad en los niños, del mismo modo que en otra época se patologizó y medicalizó la homosexualidad (proceso interesante al que ahora asistimos a su "des" patologización o "des" medicalización). Desde una óptica biomédica, la delimitación de tales problemáticas como "enfermedad" responde a la identificación de aspectos biológicos, fisiológicos, genéticos y/o neurológicos diferenciados claramente y aislados en laboratorio. En este sentido, objetivamente naturales o pertenecientes a la "naturaleza humana". Sin embargo, al analizar estos procesos "naturales" en profundidad, advertimos que muchas veces también responden a la delimitación ciertos comportamientos que es necesario dominar y controlar ya sea por considerarlos inmorales, pecaminosos o criminales $y$; al adquirir un significado médico se tornan controlables de una manera "científica": la homosexualidad o el síndrome de déficit de atención e hiperactividad en los niños entre otras categorías diagnósticas de la psiquiatría son ejemplos de ello. Cualquiera sea el caso, es difícil su adscripción a lo netamente natural o estrictamente cultural.

Si lo pensamos en términos históricos también observamos la relevancia que adquieren ciertas enfermedades vinculadas no sólo a los avances en el conocimiento científico, sino también a procesos sociales y económicos o valoraciones socioculturales. Pensemos sino en la tuberculosis en el siglo XIX durante el romanticismo, considerada síntoma de delicadeza y sensibilidad, sobresaliendo la idea "del paciente en tanto que criatura turbulenta, descuidada, de extremadas pasiones, demasiado sensible para soportar el horror del mundo cotidiano y vulgar"15. El aspecto etéreo, pálido, casi fantasmal del enfermo de tuberculosis representa a la perfección esa renuncia de lo mundano. Muchos jóvenes de buena posición coinciden en las casas de curación, adelantando una forma de vida ociosa y elitista que favorece en ocasiones el impulso creativo y que aleja a los artistas aún más de toda responsabilidad familiar o social, en una demostración literal de esa fuga de lo mundano. Este imaginario se plasma claramente en La montaña mágica, de Tomas Mann ${ }^{16}$, este autor señala: "la enfermedad es perfectamente humana, pues ser hombre es estar enfermo. En efecto, el hombre es esencialmente un enfermo, y el hecho de que esté enfermo es precisamente lo que hace de él un hombre (...) es pues del espíritu de la enfermedad de lo que depende la dignidad del hombre y su nobleza. En una palabra, es tanto más hombre cuanto más enfermo está, y el genio de la enfermedad es más humano que el genio de la salud"16. En ese proceso "existencialista" de enfermar se gesta el modelo moderno de enfermedad: el del individuo y el de su rol social como enfermo, definido por su lugar pasivo en el entramado cultural. La tuberculosis se marginaliza a medida que se evi- 
dencia su carácter contagioso, especialmente en 1866 cuando Jean Antoine Villemin demuestra que puede contagiarse la enfermedad. En la actualidad nadie puede negar el proceso orgánico que desencadena la enfermedad en las personas y el carácter biológico de la enfermedad, sin embargo tampoco puede negarse sus connotaciones sociales y que son las condiciones de vida más desfavorables que afectan a los sectores poblacionales más empobrecidos los que favorecen el contagio; y esto no es porque tengan un gen o una biología tal, sino porque las condiciones de vida favorecen el contagio y desarrollo de la enfermedad en términos biológicos. Nuevamente nos encontramos ante una problemática que se muestra como un mixto de naturaleza y cultura, un hibrido.

Podemos citar otro ejemplo como el cáncer; nadie dudaría que se trata de un proceso biológico en el que ocurre una reproducción anómala de células en el cuerpo humano, pero tampoco podemos dejar de ver, como lo analiza Horisch ${ }^{17}$, que su delimitación ocurre una época del crecimiento exponencial, ilimitado de la producción industrial, de la innovación, de las metrópolis y, como lo plantea Sontag ${ }^{15}$, que su aparición ocurre plagada de metáforas de desintegración física: el canceroso es visto como alguien a quien su propia represión emocional conduce a ese desorden máximo que es la proliferación de células malignas en el organismo.

De las metáforas asociadas con una enfermedad grave, Sontag señala una en particular: la metáfora militar. El cuerpo se concibe como un campo de batalla; el cuerpo libra frente al cáncer un combate encarnizado del que con frecuencia sale vencido. El cáncer representa el horror de una invasión generalizada, con escaramuzas imprevisibles, y terapias brutales que representan una suerte de contraofensiva militar. Es común también extender la metáfora del cuerpo enfermo a situaciones de orden político o social, hablar por ejemplo del cáncer que corroe a una sociedad o a un partido político, o la revuelta que es preciso apagar o reprimir antes que gangrene a todo el cuerpo social ${ }^{15}$. Claramente la construcción y delimitación del cáncer como enfermedad no es puramente natural.

Otro ejemplo a citar son las patologías que emergen con la proliferación del consumo, desde la reciente inclusión en el Manual diagnóstico y estadístico de los trastornos mentales ${ }^{18}$ entre los trastornos adictivos no relacionados a sustancias las compras compulsivas, hasta los trastornos alimentarios como la bulimia, anorexia u obesidad. En el caso de estos últimos, no puede negarse los efectos fisiológicos o las etiologías de base orgánica asociadas a tales patologías, sin embargo tampoco podemos dejar de ver que tales "enfermedades" responden a la instalación de ciertos modelos de belleza y éxito extraordinarios o inalcanzables, donde la delgadez extrema se convierte en el patrón, no solo en el ámbito de la moda, sino también en los medios masivos de comunicación que reproducen y potencian este discurso. Mientras, la publicidad instala un alimento de una manera que excede su valor nutricional, convirtiéndolo unas veces en icono de determinado estilo de vida, de diversión o placer, reforzando ciertos estereotipos y valores (de belleza, salud, género, entre otros) promoviendo además consumos que muchas veces no condicen con los modelos de belleza instalados.

El Antropólogo Claude Fischler ${ }^{19}$, referente en el tema de alimentación, va a plantear esta problemática en los términos dicotómicos que venimos problematizando aquí: "si se admite que, en el sistema alimentario, hay "ruido" cultural y que ese ruido es capaz de desorganizar la maquinaria biológica, los dispositivos homeostáticos internos, entonces, queda por preguntarse a través de qué procesos y mediaciones ocurre eso". Claramente aparece una base orgánica, fisiológica que tiende a la "homeostasis" y se ve afectada por el polo cultura- sociedad. Consideramos que ante estas problemáticas alimentarias, que emergen en momentos particulares de la historia y no en cualquier sociedad, sería difícil colocar el problema en lo puramente biológico o exclusivamente cultural, también es difícil delimitar ámbitos de acción o afectación de lo social hacia una base biológica, quizá sea más adecuado hablar de híbridos.

\section{Cómo ocurre el proceso de medicalización}

Hasta aquí hemos definido la medicalización, dando algunos ejemplos de estos procesos y desarrollando de qué modo los "objetos" de la medicina no son exclusivamente "objetos", sino también sujetos), cuasi-objetos o híbridos. Queda aún por indagar de qué modo esos híbridos se crean con un significado que los adscribe al campo médico. Hemos señalado que intervienen en su construcción tanto lo biológico, "natural" como lo socio-cultural, político y económico, si bien la medicina parte de la idea de estar tratando con hechos científicos.

Es en esta instancia que se torna necesario indagar sobre los procesos de producción de conocimiento científico que, especialmente en el 
campo de la medicina, ocurre mayormente en el ámbito del laboratorio. $\mathrm{Al}$ respecto es interesante el análisis de Latour y Woolgar ${ }^{20}$ en el libro "La vida en un laboratorio. La construcción de los hechos científicos" donde van a presentar el laboratorio como un sistema de inscripción literaria cuyo resultado es llevar al resto a la convicción de que algo es un hecho. Y un hecho se convierte en tal cuando pierde todo atributo o cualificación temporal y se incorpora a un cuerpo de conocimientos al que otros recurren.

Un excelente ejemplo, que desarrollaremos de manera sintética, es el llamado "Trastorno por Déficit de Atención e Hiperactividad (TDAH). Los primeros intentos por explicar el TDAH en los niños ocurren en 1935, hacia los años sesenta aparece Eisenberg, prestigioso profesor de psiquiatría en Harvard, quien vuelve sobre dicha "enfermedad", con el nombre de "reacción hipercinética de la infancia”. Con ese diagnóstico pudo tratar a alumnos "difíciles", probando diferentes psicofármacos, inicialmente dextroanfetamina y luego metilfenidato, droga que hoy en día prevalece como tratamiento y que transforma "niños enérgicos” en “dóciles”. El logro de Eisenberg fue asociar el TDAH a causas genéticas, donde puede intervenir la herencia. Así, en el año 1968 se incluyó la "reacción hipercinética de la infancia" en el Manual Diagnóstico y Estadístico (DSM) $\mathrm{y}$ desde entonces forma parte de dicho manual, sólo que ahora recibe el conocido nombre de Trastorno de Déficit de Atención e Hiperactividad (TDAH).

El mismo Eisenberg, antes de morir en el año 2009 , en una entrevista dijo que se trataba de una enfermedad inventada, que la idea de que un niño pudiera tener TDAH desde el nacimiento estaba sobrevalorada, que nunca pensó que eso pudiera tener tanta repercusión y que había que prestar más atención a lo psicosocial, al contexto y entorno familiar ${ }^{21}$. El ejemplo muestra claramente como los hechos que se construyen en laboratorio adquieren un peso que los autonomiza de otros procesos y eventos relevantes al problema, en este caso particular también tranquiliza a los padres o a los docentes, quitando responsabilidad al entorno familiar y social del niño y colocando el peso en lo biológico.

En el proceso de medicalización, no podemos dejar de ver esos hechos atravezada por la industria de los medicamentos, quién financia y qué se financia va a estar mediada por los laboratorios que producen ciertos medicamentos y necesitan la construcción de hechos científicos que avalen tal o cual tratamiento farmacológico ${ }^{22}$, pero antes también es necesario delimitar que tal o cual problema es "apto" o "necesario" de ser tratado medicamente. En esto, los medios masivos de comunicación cumplen un rol fundamental. La publicidad no solo va a instalar un medicamento, sino también una enfermedad, algunos autores como Conrad" hablan del "marketing de la enfermedad". Y en este proceso no podemos considerar a los usuarios como simples "pacientes" del sistema de salud, los usuarios reclaman la atención de ciertos padecimientos, de determinada manera, no sólo a partir de lo que la publicidad indica, sino también a partir de las múltiples fuentes de información disponibles hoy, especialmente en la web: blogs de especialistas y no especialistas, páginas de salud y revistas de divulgación científica como de detractores del conocimiento científico o posturas críticas del mismo, es con todo eso que el "paciente" llega a la consulta médica, construyendo también él en su demanda la necesidad de tratar su malestar medicamente y de determinada manera.

A modo de síntesis, el siguiente esquema (Figura 1) presenta la multiplicidad de eventos, actores, procesos que intervienen en la hibridación que caracteriza a la medicalización.

En el esquema de síntesis vemos cómo en el proceso de medicalización de las sociedades actuales intervienen diversos actores sociales:

- usuarios del sistema de salud,

- médicos

- medios masivos de comunicación,

- industria farmacéutica y

- científicos.

La producción de conocimiento científico como "hechos científicos", crea nuevas necesidades y demandas, muchas veces orientadas hacia el desarrollo de nuevos medicamentos pero también hacia la sociedad, recomendando ciertas prácticas y cuidados. En todo ello, los medios masivos de comunicación juegan un rol fundamental en la difusión de este conocimiento, especialmente a través de la publicidad, pero también en blogs, artículos de divulgación científica, columnas de opinión y cadenas de mails, disponibles cada vez más fácilmente para un público más amplio. Así, los usuarios reclaman desde una opinión formada a partir de lo observado y disponible, y el médico sería el encargado de diagnosticar, tratar y prescribir acorde a tales expectativas. Es interesante observar y analizar en este proceso, de qué modo el médico ocupa un lugar marginal respecto de su tarea central: diagnosticar y tratar -muchas veces los usuarios llegan al consultorio con un autodiagnóstico, demandando cierto tratamiento. Esto 


\section{LA HIBRIDACIÓN EN EL PROCESO DE MEDICALIZACIÓN}

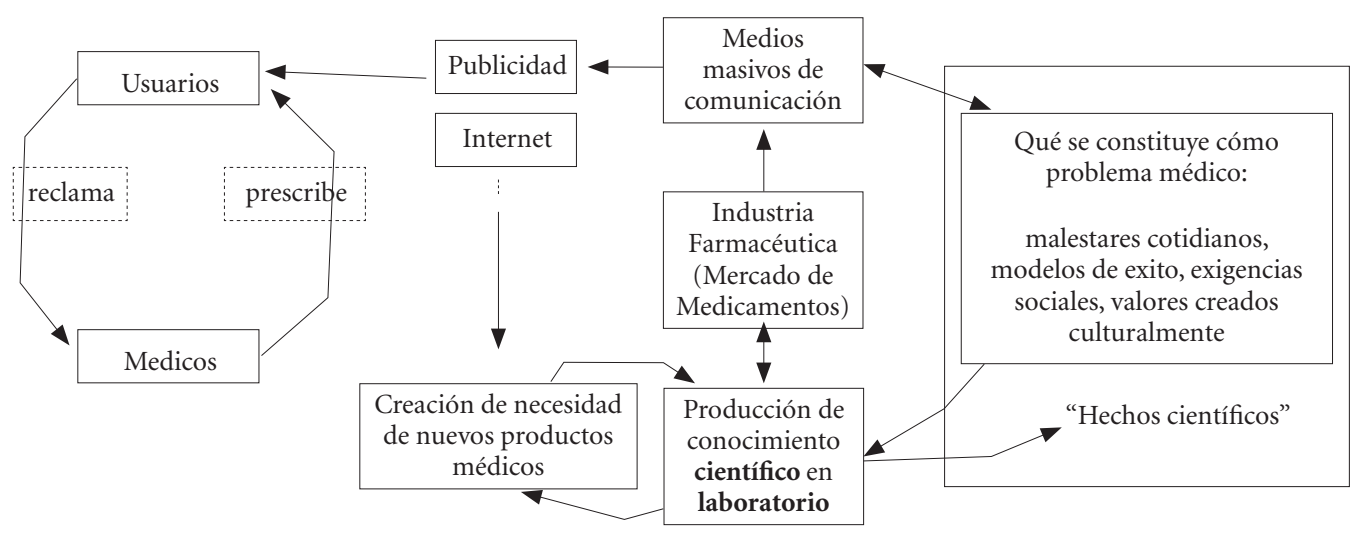

Figura 1. La hibridación em el proceso de medicalización.

es interesante para pensar que la hegemonía de la medicina, lo que Menéndez ${ }^{11,12}$ llamó el Modelo Médico Hegemónico, hoy funciona en la sociedad con gran autonomía respecto de la autoridad médica, al menos del médico en la clínica. Las personas no son simplemente "objeto" pasivo de lo que recomienda el médico, sino que también buscan deliberadamente soluciones químicas para sobrellevar malestares cotidianos. El uso y abuso de medicamentos psicotrópicos, donde no siempre media la prescripción médica, da cuenta de ello.

En relación a este proceso es interesante analizar que si bien el llamado "paciente" se convierte en "actor social" activo en relación a su salud, lo es pero a la vez expropiado de su propio saber sobre su cuerpo, su demanda no parte de un malestar que construye de la propia experiencia inter-subjetiva del padecimiento, sino que a la vez intervienen en la construcción de su demanda estas otras múltiples fuentes de "conocimiento".

El médico aparece como figura a medio camino entre la publicidad y la ciencia. Este corrimiento del lugar del médico como autoridad para dictaminar sobre la salud y enfermedad de las personas resulta novedoso. Nos permite aventurar que tal vez sea eso lo que esté ocurriendo hoy en nuestra sociedad o lo que vendrá: un médico que queda relegado en relación a la capacidad explicativa y de "seducción" que poseen otros medios como la publicidad o internet. En relación con esto, se perciben también alianzas que sería necesario analizar entre la industria quími- co farmacéutica y el desarrollo de investigaciones científicas, y de ambos con los medios; que vienen a reemplazar al médico a la hora de "vender", recomendar y avalar el uso de ciertos productos.

Esto también puede ser leído como síntoma de un malestar de la época actual, de una sociedad donde existen "medicamentos para todo", al tiempo que médicos -pensando en la hiperespecialización de la medicina-, pero además personas que buscan o desean deliberadamente ser "seducidas" por la ciencia médica para calmar sus temores, preocupaciones, dolores y ansiedades con las tecnologías que ésta ofrece. A ésta demanda también se superponen las sospechas que se alzan sobre el saber y técnicas médicas, sobre el efecto secundario o iatrogénico de los tratamientos -pensemos sino en la epidemia de Gripe A. Si bien muchos de estos malestares encuentran su causa última en las relaciones que se establecen entre las personas y de éstas con su entorno, se termina buscando atender a esos síntomas químicamente, más que a unas condiciones de vida que pueden deteriorar la salud y producir enfermedad. Nuevamente, la idea que instala la modernidad en torno a la clara distinción entre naturaleza y cultura habilita a pensar ambos procesos separadamente, tal como se presentan a los fines analíticos, cuando en realidad sería difícil dar un estatus independiente a los procesos biológicos, orgánicos o fisiológicos que desencadenan o encuentran su causa última en procesos socio-culturales tal como los hemos descripto. 


\section{Una reflexión final a modo de cierre y apertura}

La medicina ha cambiado y con ello se despierta toda clase de temores acerca de la posibilidad de la sobremedicación. El cuerpo y las enfermedades aparecen modelados conforme avanza el conocimiento médico. En este proceso ¿la medicina podría ser pensada como actuando de un modo hipocondríaco? hallando enfermedades de todo tipo en todos lados, la proliferación de "medicamentos para todo" se convierte en la herramienta diagnóstica de una segura enfermedad. En la actualidad, la publicidad ofrece todo tipo de medicamentos con una fórmula muy similar a la que se ofrece un cosmético, una hamburguesa o cigarrillos; servicios de atención a la salud e internación como si se tratara de hotelería. La salud de las personas se mercantiliza. En paralelo ocurre un creciente desarrollo científico-tecnológico que hace prescindir cada vez más del contacto directo del médico con sus pacientes.

De ello se deriva la pérdida de autoridad del médico, situándose entre la publicidad y "la ciencia" en algunos casos y, el google, en otros. La hegemonía de la biomedicina se visualiza más que nunca en el grado de consenso que adquiere en la sociedad, ya no hace falta de la figura de un médico que convenza de nada, es justo en el momento que la medicina y los médicos comienzan a discutir sobre sus excesos, sobre los efectos adversos que pueden producir muchas veces ciertos tratamientos, que la sociedad de manera autónoma reclama como necesidad y derecho la atención de padecimientos que antes formaban parte de la vida cotidiana, del ciclo vital y se resolvían en el ámbito doméstico. Los medicamentos pueden resultar tan adictivos y enfermizos como las propias enfermedades. Es en parte producto de esta "paranoia" de hoy, en la cual todo malestar puede ser objeto de tratamiento biomédico, que la vida cotidiana puede medicalizarse, y no necesariamente a causa del médico sino también de la búsqueda por escapar al dolor, a los años, al propio cuerpo y ciclo vital que marca nuestra naturaleza. Nadie niega los avances que la ciencia médica ha traído para mejorar la expectativa de vida al nacer, pero tampoco se debe perder de vista que a nivel poblacional, la calidad de vida y condiciones de vida han mejorado considerablemente más allá del desarrollo de cualquier medicamento, ese mejoramiento es lo que indudablemente produce algún impacto en la salud colectiva.

$\mathrm{El}$ análisis que presentamos habilita la discusión en torno al desarrollo de un modelo interpretativo que amplía la comprensión en torno a la hegemonía de los saberes y prácticas biomédicas y sobre los procesos de medicalización, que otorgan un lugar destacado a los profesionales de la salud. Desde el lugar crítico que ocupamos, observamos la necesidad de incluir en nuestras explicaciones respecto de la hegemonía biomédica la participación de otros actores sociales, de modo de problematizar la supuesta pasividad de los enfermos y de la población en general, así como el lugar que ocupan en la actualidad la industria químico-farmacéutica, los laboratorios, no solo en la producción, sino también en la construcción de patrones de legitimación del consumo de medicamentos (ver Silva ${ }^{22}$ y para el caso de la Gripe A Sy y Spinelli23).

En ese sentido, la crítica a la pretendida modernidad y el concepto de híbridos que propone Latour resulta fructífero para el análisis y comprensión de los procesos de medicalización de la vida en las sociedades actuales. La falsa dicotomía naturaleza/cultura no ha hecho otra cosa más que crear y reproducir la ilusión de que estamos tratando con "hechos científicos/reales", objetos que nada tienen que ver con los procesos sociales, político-económicos y culturales, lo que habilita el discurso sobre la neutralidad de ciertas intervenciones y prácticas médicas.

Volviendo sobre nuestra hipótesis, puede observarse que el discurso científico al plantear "los hechos" como tales, invisibiliza que su construcción es también un discurso social que instala una política-economía del cuidado y atención de la salud, del mismo modo que el discurso social, político-económico y cultural de la salud también es un discurso que modela los "hechos de la ciencia" y es desde este lugar que hablamos de la necesidad de pensar en términos de hibridaciones en los procesos de medicalización. 


\section{Referencias}

1. Descola P. Nature and Society: Anthropological Perspectives. Londres: Routledge; 1996.

2. Descola P. Las lanzas del crepúsculo. Relatos jíbaros. Alta amazonia. España: Fondo de Cultura Económica; 2005.

3. Descola P. Más allá de naturaleza y cultura. Buenos Aires: Amorrortu; 2012.

4. Latour B. Nunca fuimos modernos. Ensayo de antropología simétrica. Madrid: Siglo XXI; 2012.

5. Samaja J. Epistemología de la Salud. Reproducción social, subjetividad y transdisciplina. Buenos Aires: Lugar Editorial; 2004.

6. Castiel LD, Alvarez-Dardet C. La Salud Persecutoria. Los límites de la responsabilidad. Buenos Aires: Lugar Editorial; 2010.

7. Freidson E. La profesión médica. Barcelona: Península; 1978.

8. Zola I. Medicine as an institution of social control. American Sociological Review 1972; 20(4):487 504

9. Conrad P. The Medicalization of Society on the Transformation of Human Conditions into Treatable Disorders. Baltimore: Johns Hopkins University Press; 2007.

10. Illich I. Némesis Médica. Barcelona: Ed. Barral; [1973] 1975.

11. Menéndez EL. El modelo médico y la salud de los trabajadores. In: Basaglia F, compilador. La salud de los trabajadores. México: Nueva Imagen; 1978. p. 11-53.

12. Menéndez EL. Cura y Control. La apropiación de lo social por la práctica psiquiátrica. México: Nueva Imagen; 1979.

13. Conrad P. Sobre la medicalización de la anormalidad y el control social Psiquiatría Crítica. In: Ingleby D, editor. La política de la Salud Mental. Barcelona: Crítica; 1982. p. 129-154.

14. Conrad P, Schneider J. Deviance and medicalization: from badness to sickness. Philadelphia: Temple University Press; 1992.

15. Sontag S. La enfermedad y sus metáforas. El sida y sus metáforas. Buenos Aires: Taurus; 2005.

16. Mann T. La montaña mágica. Buenos Aires: Edhasa; [1924] 2009.
17. Horisch J. Las épocas y sus enfermedades. El saber patognóstico de la literatura. In: Bongers W, Olbrich T, organizadores. Literatura, Cultura, Enfermedad. Buenos Aires: Paidós; 2006. p. 5-30.

18. American Psychiatric Association (APA). Diagnostic and Statistical Manual of Mental Disorders (DSM-5). $5^{\text {th }}$ ed. Washington: APA; 2013.

19. Fischler C. Gastro-nomia y gastro-anomia. Sabiduria del cuerpo y crisis biocultural de la alimentación moderna. Gazeta de Antropología 2010; 26(1):1-19.

20. Latour B, Woolgar S. La vida en el laboratorio. La construcción de los hechos científicos. Madrid: Alianza; 1995.

21. Von Blech J. Schwermut ohne Scham. Der Spiegel 2012; 6: 122-131.

22. Silva CD. Por uma filosofia do medicamento. Cien Saude Colet 2015; 20 (9):2813-2824.

23. Sy A, Spinelli H. Dimensiones políticas de una epidemia: El caso de la gripe A (H1N1) en la prensa escrita. Cad Saude Publica 2016; 32(3):1-11.

Artigo apresentado em 13/04/2016

Aprovado em 20/07/2016

Versão final apresentada em 22/07/2016 